\title{
Evaluation of different nested PCRs for detection of Anaplasma phagocytophilum in ruminants and ticks
}

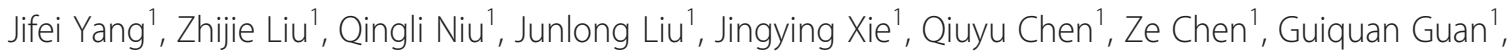
Guangyuan Liu', Jianxun Luo ${ }^{1 *}$ and Hong Yin ${ }^{1,2^{*}}$

\begin{abstract}
Background: Anaplasma phagocytophilum is a causative agent of granulocytic anaplasmosis in mammals, which has a broad geographical distribution and a high degree of clinical diversity. Currently, numerous PCR assays have been developed and used for the detection of A. phagocytophilum in various specimens. However, their performance varies. The aim of this study was to evaluate the performance of five nested PCR assays by detection of 363 ruminant and tick samples, and to select the most appropriate methods for the sensitive detection of A. phagocytophilum in environmental or clinical samples.
\end{abstract}

Results: Positive PCR results for A. phagocytophilum were obtained in 75 (20.7 \%), 42 (11.6\%) and 19 (5.2\%) specimens with primer sets EC (EC9/EC12a and SSAP2f/SSAP2r), EE (EE1/EE2 and EE3/EE4) and ge (ge3a/ge10r, ge9f/ge2), respectively. The amplification of template DNA with the primer set MSP (MAP4AP5/MSP4AP3, msp4f/msp4r) could not be obtained in both ruminants and ticks, and a low specificity of the EL primers [EL(569)F/EL(1193)R, EL(569)F/ EL(1142)R] in tick samples was observed. Our results revealed that the nested PCR with primer set EC complementary to the 165 rRNA gene was the most sensitive assay for detection of A. phagocytophilum in ruminant and tick specimens. A. phagocytophilum was detected in 47 (35.1\%) sheep, 12 (10.4\%) cattle, and 17 (14.9\%) ticks. Two A. phagocytophilum genotypes were identified, that varied between sheep and cattle in sample collection sites.

Conclusions: This report provides more valuable information for the diagnosis and management of granulocytic anaplasmosis in China.

Keywords: A. phagocytophilum, Diagnosis, PCR, Ruminants, Tick, Prevalence

\section{Background}

HGA (Human granulocytic anaplasmosis) is an emerging tick-borne zoonosis caused by the obligate intracellular bacterium Anaplasma phagocytophilum (formerly known as Ehrlichia phagocytophila, Ehrlichia equi or the HGE agent) $[1,2]$. The organism is commonly maintained in nature through an enzootic cycle involving ticks and vertebrate hosts [3, 4]. Several Ixodes ticks are known or suspected vectors of $A$. phagocytophilum, including Ixodes scapularis and Ixodes pacificus in North America, Ixodes

\footnotetext{
*Correspondence: luojianxun@caas.cn; yinhong@caas.cn

'State Key Laboratory of Veterinary Etiological Biology, Key Laboratory of Veterinary Parasitology of Gansu Province, Lanzhou Veterinary Research Institute, Chinese Academy of Agricultural Science, Xujiaping 1, Lanzhou, Gansu 730046, P. R. China

Full list of author information is available at the end of the article
}

ricinus in Europe, and Ixodes persulcatus in Russia and Asian [3, 5-8]. A. phagocytophilum infects a variety of hosts and causes granulocytic anaplasmosis in humans but also in wild and domestic animals [9, 10]. Since the first suspected human case was reported in Anhui province in 2006, more than 90 cases of HGA have been recorded in Beijing, Tianjin, Shandong, Henan, Hubei and Inner Mongolia in China [11, 12]. The actual number of human cases may be much higher due to the poor diagnostic tools, non-specificity of the reported symptoms and lack of awareness of public health professionals [13].

Rapid and sensitive detection of A. phagocytophilum is an essential step for the control and prevention of HGA in endemic areas. A. phagocytophilum was initially 
identified as a human agent using molecular methods rather than culture or serological tests $[1,14]$. Since then, PCR assays have played an important role in the laboratory diagnosis of HGA in clinical and environmental specimens for their rapidity and relative ease of performance. However, their performance varies significantly $[15,16]$. Most studies have always focused on the analytical sensitivity or specificity of those PCR assays, and the ability to detect small amounts of nucleic acid and specific nucleic acid fragments, to enable distinction of closely related strains $[15,16]$. However, considerable variation within $A$. phagocytophilum strains has been described, and isolates from various hosts or geographic locations have displayed genetic diversity and divergence within frequently used PCR-target genes, such as groESL, ankA and msp4 [17-19]. Some assays could not detect all the variants or ecotypes of $A$. phagocitophilum. Thus, it is critical to evaluate the performance of prospective assays in certain species and in a given geographic area in order to obtain more reliable results. The objective of this study was to evaluate five nested PCRs for detection of A. phagocytophilum in ruminants and tick specimens from northwest China.

\section{Methods}

EDTA- $\mathrm{K}^{2+}$ anticoagulated blood samples were taken from the jugular vein of 249 asymptomatic domestic ruminants (134 sheep and 115 cattle) and collected in a sterile tube in May 2015 from Ili Kazakh Autonomous Prefecture, in northern Xinjiang, China. One hundred and fourteen ticks were collected from sheep, cattle and other livestock within same herds. Five species of ticks were identified in accordance with the standard taxonomic keys [20]. Seventy-two ticks collected from cattle were identified as Dermacentor marginatus; 35 ticks collected from sheep were identified as Haemaphysalis punctata $(n=28)$, Haemaphysalis concinna $(n=3)$ and Hyalomma asiaticum $(n=4)$; and seven ticks collected from horses were identified as Hyalomma detritum. The samples collection in the present study was consented by animals owners. All animal treatments and handling complied with Ethical Guidelines and were approved by the Animal Ethics Committee of Lanzhou Veterinary Research Institute, Chinese Academy of Agricultural Sciences.

DNA was extracted from blood and tick samples using a Gentra Puregene DNA Purification kit (Qiagen) as described previously [9]. All DNA samples were examined for the presence of $A$. phagocytophilum by nested PCRs. The PCR primers are listed in Table 1 . The reaction was performed in an automatic thermocycler (Bio-Rad, Hercules, CA, USA) in a total volume of $25 \mu \mathrm{L}$ containing $2.5 \mu \mathrm{L}$ of $10 \times$ PCR buffer $\left(\mathrm{Mg}^{2+}\right.$ Plus), $2.0 \mu \mathrm{L}$ of each dNTP at $2.5 \mathrm{mM}, 1.25 \mathrm{U}$ of Taq DNA polymerase

Table 1 Oligonucleotide primers used for detection of A. phagocutophilum

\begin{tabular}{|c|c|c|c|c|c|}
\hline Target gene & Primer name & Primer Sequence $\left(5^{\prime}-3^{\prime}\right)$ & Annealing temp $\left({ }^{\circ} \mathrm{C}\right)$ & Amplicon size (bp) & Reference \\
\hline \multirow[t]{4}{*}{165 rRNA } & ge3a & CACATGCAAGTCGAACGGATTATTC & 55 & 932 & [25] \\
\hline & ge10r & TTCCGTTAAGAAGGATCTAATCTCC & & & \\
\hline & gegf & AACGGATTATTCTTTATAGCTTGCT & 55 & 546 & \\
\hline & ge2 & GGCAGTATTAAAAGCAGCTCCAGG & & & \\
\hline \multirow[t]{4}{*}{ Msp4 } & MAP4AP5 & ATGAATTACAGAGAATTGCTTGTAGG & 54 & 849 & {$[24,27]$} \\
\hline & MSP4AP3 & TTAATTGAAAGCAAATCTTGCTCCTATG & & & \\
\hline & msp4f & CTATTGGYGGNGCYAGAGT & 55 & 381 & \\
\hline & msp4r & GTTCATCGAAAATTCCGTGGTA & & & \\
\hline \multirow[t]{4}{*}{$16 S$ rRNA } & $\mathrm{EE}-1$ & TCCTGGCTCAGAACGAACGCTGGCGGC & 50 & 1433 & [23] \\
\hline & $\mathrm{EE}-2$ & AGTCACTGACCCAACCTTAAATGGCTG & & & \\
\hline & $\mathrm{EE}-3$ & GTCGAACGGATTATTCTTTATAGCTTGC & 50 & 926 & \\
\hline & $\mathrm{EE}-4$ & CCCTTCCGTTAAGAAGGATCTAATCTCC & & & \\
\hline \multirow[t]{4}{*}{ groEL } & EL(569)F & ATGGTATGCAGTTTGATCGC & 62 & 624 & {$[26,34]$} \\
\hline & EL(1193)R & TCTACTCTGTCTTTGCGTTC & & & \\
\hline & $\mathrm{EL}(569) \mathrm{F}$ & ATGGTATGCAGTTTGATCGC & 56 & 573 & \\
\hline & $\mathrm{EL}(1142) \mathrm{R}$ & TTGAGTACAGCAACACCACCGGAA & & & \\
\hline \multirow[t]{4}{*}{165 rRNA } & EC9 & TACCTTGTTACGACTT & 55 & 1462 & [35] \\
\hline & $\mathrm{EC} 12 \mathrm{a}$ & TGATCCTGGCTCAGAACGAACG & & & \\
\hline & SSAP2f & GCTGAATGTGGGGATAATTTAT & 55 & 641 & \\
\hline & SSAP2r & ATGGCTGCTTCCTITCGGTTA & & & \\
\hline
\end{tabular}


(TaKaRa, Dalian, China), $2.0 \mu \mathrm{L}$ of template DNA, $1.0 \mu \mathrm{L}$ of each primer $(20 \mathrm{pmol})$ and $16.25 \mu \mathrm{L}$ of distilled water. DNA from sheep infected with $A$. phagocytophilum validated by sequencing (Gene accession no. JN558811) was used as a positive control, and sterile water was used as the blank control for each run. The cycling conditions for the first and second round amplification involved $4 \mathrm{~min}$ of denaturation at $94{ }^{\circ} \mathrm{C}, 35$ cycles at $94{ }^{\circ} \mathrm{C}$ for $30 \mathrm{~s}$, annealing for $30 \mathrm{~s}$ at a temperature dependent on the primers applied (annealing temperatures shown in Table 1), and $72{ }^{\circ} \mathrm{C}$ for 1 to $1.5 \mathrm{~min}$ (dependent on the length of target fragments), with a final extension step at $72{ }^{\circ} \mathrm{C}$ for $10 \mathrm{~min}$. The PCR products were visualized by UV transillumination in a $1.5 \%$ agarose gel following electrophoresis and staining with ethidium bromide.

A. phagocytophilum positive samples were selected randomly and verified by sequencing. The PCR products were cloned into the pGEM-T Easy vector (Promega, Shanghai, China) and subjected to bidirectional sequencing (Sangon Biotech, Shanghai, China). The sequences obtained were compared with reference sequences from GenBank (see Additional file 1). A phylogenetic tree was then constructed using the neighbor-joining (NJ) algorithm with the Kimura two-parameter model of the Mega 4.0 Software [21]. The GenBank accession numbers for the partial 16S rRNA gene sequences obtained in this study were as follows: KT944028-KT944029 and KT951192.

The results were analyzed using a Chi-square test in Predictive for Analytics Software (PASW) Statistics version 18. A difference was considered statistically significant at $P<0.05$.

\section{Results}

Of the total 363 ruminant and tick specimens that were included in our evaluation of the five nested PCR assays, positive PCR results for $A$. phagocytophilum were obtained in 75 (20.7\%), 42 (11.6\%) and 19 (5.2\%) specimens with primer sets EC (EC9/EC12a and SSAP2f/SSAP2r), EE (EE1/EE2 and EE3/EE4) and ge (ge3a/ge10r, ge9f/ge2), respectively (Table 2). DNA of A. phagocytophilum was found in only two cattle specimens using primer set EL [EL(569)F/EL(1193)R, EL(569)F/EL(1142)R]. However, unspecific products were obtained in tick specimens with EL primers as a result of two bands between 250 and $500 \mathrm{bp}$ (data not shown). Under the PCR conditions outlined, amplification of the template DNA with the primer set MSP (MAP4AP5/MSP4AP3, msp4f/msp4r) could not be obtained (Table 2). Apart from the primer sets EL and MSP, the assays showed that the positive rates of $A$. phagocytophilum infection in ruminant and tick specimens ranged from 5.6 to $20.7 \%$. The highest positive rate $(20.7 \%, 75 / 363)$ was observed using the EC primer set (Table 2). The PCR with EC primer set was more sensitive than the ones with $\mathrm{EE}$ and ge primer sets $(\mathrm{Chi} 2=39.944$, $\mathrm{df}=2, P<0.001)$; and the PCR with EE primer set was more sensitive than that with ge primer set $(\mathrm{Chi} 2=9.468$, $\mathrm{df}=1, P<0.01)$. Moreover, an additional tick sample (2-21) was negative with EC primers but positive with EE, which gave an overall positivity rate of $20.9 \%$ in our sample population. As shown in Table 2, the infection rates of $A$. phagocytophilum were $35.1 \%, 10.4 \%$ and $14.9 \%$ in sheep, cattle and ticks, respectively. Of those tick samples, A. phagocytophilum was detected in $D$. marginatus collected from cattle and $H$. punctata collected from sheep.

The specificity of the assay was controlled by sequencing. Considering the highest sensitivity of the assay with EC primers in this study, 20 samples (four from cattle, seven from sheep, five from $D$. marginatus and four from $H$. punctata) positive for EC primers and the tick sample (D. marginatus, sample ID: 2-21, negative for EC but positive for EE) were selected for sequencing. Sequence analyzed by a BLASTn search in GenBank confirmed the presence of $A$. phagocytophilum in those samples. Four sequences (GenBank accession no. KT944028) identified in cattle showed 99.7 \% identity to the isolate KS20 (GenBank accession no. KJ782390) from cattle in Kashgar, Xinjiang province. The remaining 16 sequences (GenBank accession no. KT944029) identified in sheep and ticks were identical to each other and showed $99.2 \%$ identity to strain YC38

Table 2 A. phagocytophilum in ruminants and ticks detected by nested PCRs

\begin{tabular}{|c|c|c|c|c|c|c|}
\hline \multirow{2}{*}{$\begin{array}{l}\text { Host } \\
\text { (No. tested) }\end{array}$} & \multicolumn{6}{|c|}{ No. (\%) positive with: } \\
\hline & $\begin{array}{l}\text { EC9/EC12a } \\
\text { SSAP2f/SSAP2r }\end{array}$ & $\begin{array}{l}\mathrm{EE} 1 / \mathrm{EE} 2 \\
\mathrm{EE} 3 / \mathrm{EE} 4\end{array}$ & $\begin{array}{l}\text { ge3a/ge10r } \\
\text { ge9f/ge2 }\end{array}$ & $\begin{array}{l}\text { EL(569)F/EL(1 193)R } \\
\text { EL(569)F/EL(1142)R }\end{array}$ & $\begin{array}{l}\text { MAP4AP5/MSP4AP3 } \\
\text { msp4f/msp4r }\end{array}$ & $\begin{array}{l}\text { At least } \\
\text { one primer }\end{array}$ \\
\hline $\begin{array}{l}\text { Sheep } \\
(n=134)\end{array}$ & $47(35.1)$ & $31(23.1)$ & $9(6.7)$ & $0(0)$ & $0(0)$ & $47(35.1)$ \\
\hline $\begin{array}{l}\text { Cattle } \\
(n=115)\end{array}$ & $12(10.4)$ & $6(5.2)$ & $3(2.6)$ & $2(1.7)$ & $0(0)$ & $12(10.4)$ \\
\hline $\begin{array}{l}\text { Tick } \\
(n=114)\end{array}$ & $16(14)$ & $5(4.4)$ & $7(6.1)$ & $N A^{a}$ & $0(0)$ & $17(14.9)$ \\
\hline $\begin{array}{l}\text { Total } \\
(n=363)\end{array}$ & $75(20.7)$ & 42 (11.6) & $19(5.2)$ & $N A^{a}$ & $0(0)$ & 76 (20.9) \\
\hline
\end{tabular}

a not applied 
(GenBank accession no. KJ782381) from sheep in Yecheng, Xinjiang province. Moreover, one sequence (GenBank accession no. KT951192) identified in D. marginatus tick (2-21) had 98.9 \% identity to the BL102-7 strain (GenBank accession no. KJ410249) derived from Hyalomma asiaticum in Xinjiang. Phylogenetic analyses revealed that the $A$. phagocytophilum isolates identified in this study are placed on two separate clades (Fig. 1).

\section{Discussion}

A. phagocytophilum is an emerging tick-borne zoonotic agents of public health significance [1]. The disease presents as a clinical syndrome, ranging from asymptomatic to fatal disease [22]. Nonspecific symptoms and signs are manifested in the disease state, and most commonly manifested by fever, chills, headache, and myalgias, which are difficult to differentiate from those of other febrile illness $[15,22]$. PCR-based methods are powerful tools and play an important role in the confirmation of A. phagocytophilum infection in clinical and environmental specimens. Since the first identification of the HGA agent in 1994, numerous PCR amplification assays and primer sets have been described for detection of $A$. phagocytophilum [1, 23-27]. However, their performance varies [16, 28]. Thus, a choice of PCR methods with appropriate primers that target different DNA segments of A. phagocytophilum is crucial for obtaining the best possible results, and this affects the sensitivity and specificity of the diagnostic assays significantly.

A. phagocytophilum infection has been reported in humans, wild and domestic animals in China, and the infection rates were variable in different hosts or geographic locations $[9,12,29,30]$. In this study, the positive rate was significantly higher in sheep than in cattle $($ Chi $2=20.781, \mathrm{df}=1, P<0.001)$, similar result has been described in previous report [29]. Of those tick samples, A. phagocytophilum infection was found in $D$. marginatus and $H$. punctata ticks. However, we could not conclude that the role of $D$. marginatus and $H$. punctata as reservoirs or vectors of $A$. phagocytophilum because of the agent detected in this study could be from the hosts, which warrants further investigation.

In the present study, five primer sets, designed to amplify $16 S r R N A$, the heat shock gene operon groESL and major surface protein gene $m s p 4$, were used to evaluate the applicability of nested PCR for detection of A. phagocytophilum DNA in ruminant and tick specimens. Considerable differences were observed in the performance of these five assays. The results indicated that the nested PCR with EC primer set targeting the $16 \mathrm{~S}$ rRNA gene is the most sensitive method $(\mathrm{Chi} 2=$ $39.944, \mathrm{df}=2, P<0.001)$. The assay with MSP primer set appeared less useful for detection of A. phagocytophilum in ruminants and ticks, and the EL primer set was less effective for detection of tick specimens. The primer sets EE was more sensitive than ge, MSP and EL, but significantly less useful than EC (Chi2 $=11.096, \mathrm{df}=1$, $P<0.01)$. However, a previous evaluation of several different published methods for PCR amplification of specific DNA in A. phagocytophilum-infected HL-60 cells showed that the ge primer set provided the highest analytical sensitive and specificity, which could detect the equivalent of 0.25 infected cells [16]. Beyond the optimization of the nucleic acid amplification conditions, the PCR amplification efficiency could be influenced by the concentration of DNA templates in this

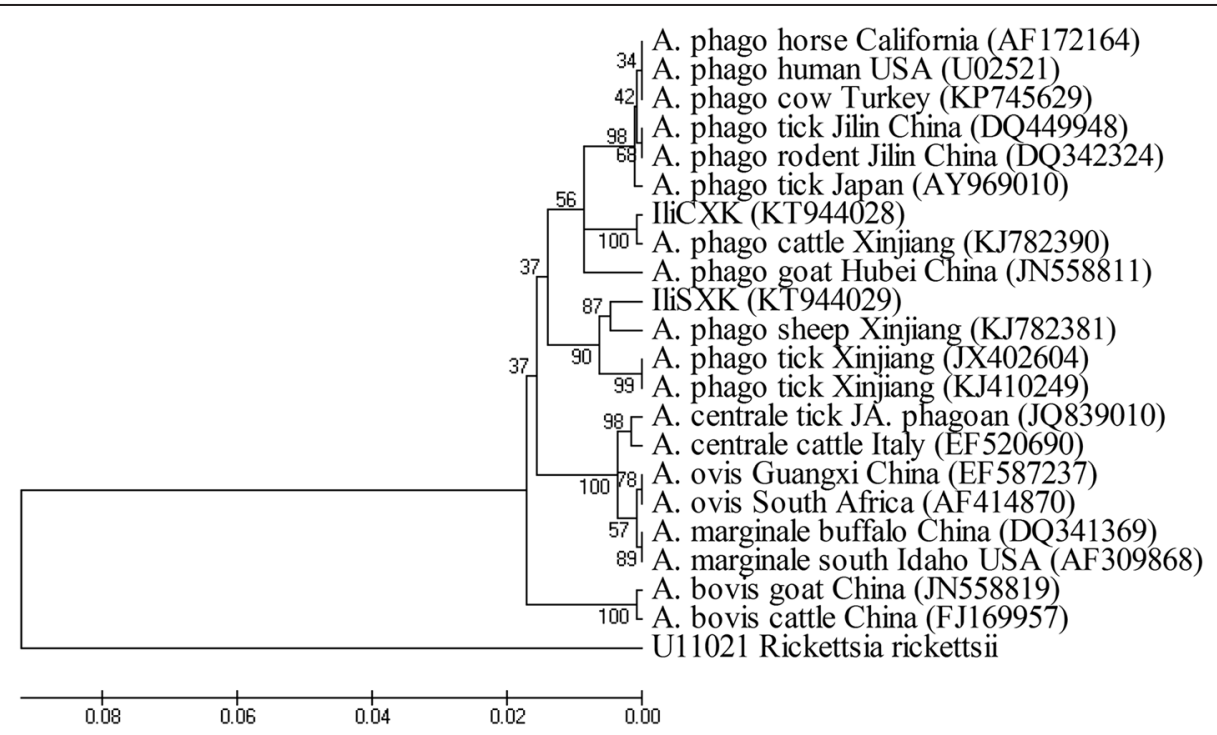

Fig. 1 Phylogenetic analysis of A. phagocytophilum (A. phago) based on 165 rRNA gene partial sequences (599 bp). Rickettsia rickettsii is used as an outgroup 
comparative analysis study. Although one tick sample (2-21) was negative for EC but positive for EE, the assay with the EC primer set showed high sensitivity in ruminants and ticks. Sequence analysis of EC-positive samples verified the specificity of the assay. Based on the comparative analysis of those five assays, the nested PCR with EC primer set would provide more reliable results for $A$. phagocytophilum detection in ruminant and tick specimens.

Phylogenetic analysis of the obtained A. phagocytophilum 16S rRNA gene sequences in this study showed that they were variable from the sequence from the positive control (Gene accession no. JN558811), excluded possible contamination and indicated the complexity of the genotype of the A. phagocytophilum in the field (Fig. 1). The A. phagocytophilum strain one (GenBank accession no. KT944028) identified in cattle was most closely related to the isolate detected in cattle from Kashgar (GenBank accession no. KJ782390) (Fig. 1). The strain two (GenBank accession no. KT944029) identified in sheep and ticks were most closely related to the isolate detected in sheep from Yecheng (GenBank accession no. KJ782381) (Fig. 1). These results suggested that $A$. phagocytophilum genotypes are vary between sheep and cattle in sample collection sites.

The results of the present study support the use of nested PCR with primer set EC (EC9/EC12a and SSAP2f/ SSAP2r) targeting the 16S rRNA gene, which was the most sensitive assay for the detection of $A$. phagocytophilum DNA in ruminants and ticks in the region investigated in China. Although this assay was more sensitive than others, it could also miss positive samples for unknown reasons. Given that no test is actually $100 \%$ sensitive or specific, an important consideration is that two or more assays should always be used in parallel to achieve maximum sensitivity for the molecular detection of A. phagocytophilim.

There are numerous factors involved in the optimization of PCR assays, and the potential discrepancies make it difficult to ensure the performance of a given assay in different laboratories. A. phagocytophilum displayed a high degree of genetic diversity, host tropisms and variation in pathogenicity [31]. Considerable strain variation of $A$. phagocytophilum has been reported in different hosts or geographic locations, and the organism can be genetically divided into several subclusters using groESL, ankA and msp4 [17-19]. Recently, four distinct ecological clusters correlate with host species have been established based on ankA gene [32], four geographically dispersed ecotypes were identified based on groESL gene and showed significantly different host ranges in Europe [33]. Thus, each laboratory should determine the efficacy of those assays under local conditions, and the choice of appropriate assays could yield accurate results for the detection of $A$. phagocytophilum in ticks and animals.

\section{Conclusions}

The performance of five nested PCR assays was accessed by parallel detection of field-collected samples. The nested PCR with primer set EC (EC9/EC12a and SSAP2f/SSAP2r) targeting the $16 S$ rRNA gene was the most sensitive assay for the detection of $A$. phagocytophilum DNA in ruminants and ticks. A. phagocytophilum was detected in 47 (35.1\%) sheep, 12 (10.4\%) cattle, and 17 (14.9\%) ticks collected from Ili Kazakh Autonomous Prefecture in northern Xinjiang, and two A. phagocytophilum genotypes were identified. These findings not only provide valuable information for the control of $A$. phagocytophilum infection, but also indicate potential implications for public health.

\section{Additional file}

Additional file 1: Table S1. Nucleotide sequence accession numbers of A. phagocytophilum, A. centrale, A. ovis, A. marginale, A. bovis, Anaplasma $\mathrm{sp}$. and $R$. rickettsii isolates analyzed in this study. (DOCX $35 \mathrm{~kb}$ )

\section{Competing interests}

The authors declare that they have no competing interests.

\section{Authors' contributions}

HY and JY designed this study and critically revised the manuscript. $J \mathrm{~L}$ participated in its design, coordination and manuscript revision. JY, ZL, QC, ZC and QN participated in sample collection and identification. JY, JX, $J$, GL and GG performed the experiments, data analysis, and drafted the manuscript. All authors read and approved the final manuscript.

\section{Acknowledgements}

This study was financially supported by the NSFC (№31372432, №31201899, №31272556, №31402189, №31471967), ASTIP, FRIP (2014ZL010), CAAS;

Creative Research Groups of Gansu Province (№1210RJIA006); NBCIS CARS-38, Special Fund for Agro-scientific Research in the Public Research (№201303035, №201303037), MOA; 973 Program (2015CB150300), Supporting Program (2013BAD12B03, 2013BAD12B05), MOST, China; Jiangsu Co-innovation Center programme for Prevention and Control of Important Animal Infectious Diseases and Zoonoses, State Key Laboratory of Veterinary Etiological Biology Project. The research was also facilitated by CRP No. 16198/RO IAEA.

\section{Author details}

${ }^{1}$ State Key Laboratory of Veterinary Etiological Biology, Key Laboratory of Veterinary Parasitology of Gansu Province, Lanzhou Veterinary Research Institute, Chinese Academy of Agricultural Science, Xujiaping 1, Lanzhou, Gansu 730046, P. R. China. ${ }^{2}$ Jiangsu Co-innovation Center for Prevention and Control of Important Animal Infectious Diseases and Zoonoses, Yangzhou 225009, P. R. China.

Received: 8 September 2015 Accepted: 18 February 2016

Published online: 24 February 2016

\section{References}

1. Chen SM, Dumler JS, Bakken JS, Walker DH. Identification of a granulocytotropic Ehrlichia species as the etiologic agent of human disease. J Clin Microbiol. 1994; 32(3):589-95.

2. Zhan L, Cao WC, Chu CY, Jiang BG, Zhang F, Liu W, Dumler JS, Wu XM, Zuo SQ, Zhang PH. Tick-borne agents in rodents, China, 2004-2006. Emerg Infect Dis. 2009;15(12):1904.

3. Bown KJ, Begon M, Bennett M, Woldehiwet Z, Ogden NH. Seasonal dynamics of Anaplasma phagocytophila in a rodent-tick (Ixodes trianguliceps) system, United Kingdom. Emerg Infect Dis. 2003;9(1):63-70.

4. Dumler JS, Barbet AF, Bekker CP, Dasch GA, Palmer GH, Ray SC, Rikihisa Y, Rurangirwa FR. Reorganization of genera in the families Rickettsiaceae and 
Anaplasmataceae in the order Rickettsiales: unification of some species of Ehrlichia with Anaplasma, Cowdria with Ehrlichia and Ehrlichia with Neorickettsia, descriptions of six new species combinations and designation of Ehrlichia equi and 'HGE agent' as subjective synonyms of Ehrlichia phagocytophila. Int J Syst Evol Microbiol. 2001;51(Pt 6):2145-65.

5. Cao WC, Zhao QM, Zhang PH, Yang H, Wu XM, Wen BH, Zhang XT, Habbema JD. Prevalence of Anaplasma phagocytophila and Borrelia burgdorferi in Ixodes persulcatus ticks from northeastern China. Am J Trop Med Hyg. 2003;68(5):547-50.

6. Liz JS, Anderes L, Sumner JW, Massung RF, Gern L, Rutti B, Brossard M. PCR detection of granulocytic ehrlichiae in Ixodes ricinus ticks and wild small mammals in western Switzerland. J Clin Microbiol. 2000;38(3):1002-7.

7. Shpynov S, Fournier PE, Rudakov N, Tarasevich I, Raoult D. Detection of members of the genera Rickettsia, Anaplasma, and Ehrlichia in ticks collected in the Asiatic part of Russia. Ann N Y Acad Sci. 2006;1078:378-83.

8. Telford 3rd SR, Dawson JE, Katavolos P, Warner CK, Kolbert CP, Persing DH Perpetuation of the agent of human granulocytic ehrlichiosis in a deer tickrodent cycle. Proc Natl Acad Sci U S A. 1996;93(12):6209-14.

9. Yang J, Liu Z, Guan G, Liu Q, Li Y, Chen Z, Ma M, Liu A, Ren Q, Luo J, et al. Prevalence of Anaplasma phagocytophilum in ruminants, rodents and ticks in Gansu, north-western China. J Med Microbiol. 2013:62(Pt 2):254-8.

10. Foley JE, Nieto NC, Adjemian J, Dabritz H, Brown RN. Anaplasma phagocytophilum infection in small mammal hosts of Ixodes ticks, western United States. Emerg Infect Dis. 2008;14(7):1147-50.

11. Zhang L, Liu Y, Ni D, Li Q, Yu Y, Yu XJ, Wan K, Li D, Liang G, Jiang X, et al. Nosocomial transmission of human granulocytic anaplasmosis in China. JAMA. 2008;300(19):2263-70.

12. Fang LQ, Liu K, Li XL, Liang $S$, Yang $Y$, Yao HW, Sun RX, Sun Y, Chen WJ, Zuo SQ, et al. Emerging tick-borne infections in mainland China: an increasing public health threat. Lancet Infect Dis. 2015;15(12):1467-79.

13. Jin H, Wei F, Liu Q, Qian J. Epidemiology and control of human granulocytic anaplasmosis: a systematic review. Vector Borne Zoonotic Dis. 2012;12(4): 269-74

14. Bakken JS, Dumler JS, Chen SM, Eckman MR, Van Etta LL, Walker DH. Human granulocytic ehrlichiosis in the upper Midwest United States. A new species emerging? JAMA. 1994;272(3):212-8.

15. Dumler JS, Brouqui P. Molecular diagnosis of human granulocytic anaplasmosis. Expert Rev Mol Diagn. 2004;4(4):559-69.

16. Massung RF, Slater KG. Comparison of PCR assays for detection of the agent of human granulocytic ehrlichiosis, Anaplasma phagocytophilum. J Clin Microbiol. 2003;41(2):717-22.

17. Morissette E, Massung RF, Foley JE, Alleman AR, Foley P, Barbet AF. Diversity of Anaplasma phagocytophilum strains, USA. Emerg Infect Dis. 2009;15(6): 928-31.

18. Strasek Smrdel K, Tozon N, Duh D, Petrovec M, Avsic Zupanc T. Diversity of groESL sequences of Anaplasma phagocytophilum among dogs in Slovenia. Clin Microbiol Infect. 2009;15 Suppl 2:79-80.

19. Strasek Smrdel K, von Loewenich FD, Petrovec M, Avsic Zupanc T. Diversity of ankA and msp4 genes of Anaplasma phagocytophilum in Slovenia. Ticks Tick Borne Dis. 2015;6(2):164-6.

20. Deng GF, Jiang ZL. Economic insect fauna of China, Fasc 39 Acari: Ixodiate. Beijing: Science Press; 1991

21. Tamura K, Dudley J, Nei M, Kumar S. MEGA4: Molecular Evolutionary Genetics Analysis (MEGA) software version 4.0. Mol Biol Evol. 2007;24(8):1596-9.

22. Bakken JS, Dumler JS. Clinical diagnosis and treatment of human granulocytotropic anaplasmosis. Ann N Y Acad Sci. 2006;1078:236-47.

23. Barlough JE, Madigan JE, DeRock E, Bigornia L. Nested polymerase chain reaction for detection of Ehrlichia equi genomic DNA in horses and ticks (Ixodes pacificus). Vet Parasitol. 1996:63(3-4):319-29.

24. Bown KJ, Lambin X, Ogden NH, Petrovec M, Shaw SE, Woldehiwet Z, Birtles RJ. High-resolution genetic fingerprinting of European strains of Anaplasma phagocytophilum by use of multilocus variable-number tandem-repeat analysis. J Clin Microbiol. 2007;45(6):1771-6.

25. Massung RF, Slater K, Owens JH, Nicholson WL, Mather TN, Solberg VB, Olson JG. Nested PCR assay for detection of granulocytic ehrlichiae. J Clin Microbiol. 1998;36(4):1090-5

26. Reye AL, Hubschen JM, Sausy A, Muller CP. Prevalence and seasonality of tick-borne pathogens in questing Ixodes ricinus ticks from Luxembourg. Appl Environ Microbiol. 2010;76(9):2923-31.
27. de la Fuente J, Massung RF, Wong SJ, Chu FK, Lutz H, Meli M, von Loewenich FD, Grzeszczuk A, Torina A, Caracappa S, et al. Sequence analysis of the msp4 gene of Anaplasma phagocytophilum strains. J Clin Microbiol. 2005;43(3):1309-17.

28. Schotthoefer AM, Meece JK, Ivacic LC, Bertz PD, Zhang K, Weiler T, Uphoff TS, Fritsche TR. Comparison of a real-time PCR method with serology and blood smear analysis for diagnosis of human anaplasmosis: importance of infection time course for optimal test utilization. J Clin Microbiol. 2013;51(7):2147-53.

29. Yang J, Li Y, Liu Z, Liu J, Niu Q, Ren Q, Chen Z, Guan G, Luo J, Yin H. Molecular detection and characterization of Anaplasma spp. in sheep and cattle from Xinjiang, northwest China. Parasit Vectors. 2015;8:108.

30. Liu Z, Ma M, Wang Z, Wang J, Peng Y, Li Y, Guan G, Luo J, Yin H. Molecular survey and genetic identification of Anaplasma species in goats from central and southern China. Appl Environ Microbiol. 2012;78(2):464-70.

31. Barakova I, Derdakova M, Carpi G, Rosso F, Collini M, Tagliapietra V, Rampon C, Hauffe HC, Rizzoli A. Genetic and ecologic variability among Anaplasma phagocytophilum strains, northern Italy. Emerg Infect Dis. 2014;20(6):1082-5.

32. Scharf W, Schauer S, Freyburger F, Petrovec M, Schaarschmidt-Kiener D, Liebisch G, Runge M, Ganter M, Kehl A, Dumler JS, et al. Distinct host species correlate with Anaplasma phagocytophilum ankA gene clusters. J Clin Microbiol. 2011:49(3):790-6.

33. Jahfari S, Coipan EC, Fonville M, van Leeuwen AD, Hengeveld $P$, Heylen D, Heyman P, van Maanen C, Butler CM, Foldvari G, et al. Circulation of four Anaplasma phagocytophilum ecotypes in Europe. Parasit Vectors. 2014;7:365.

34. Alberti A, Zobba R, Chessa B, Addis MF, Sparagano O, Pinna Parpaglia ML, Cubeddu T, Pintori G, Pittau M. Equine and canine Anaplasma phagocytophilum strains isolated on the island of Sardinia (Italy) are phylogenetically related to pathogenic strains from the United States. Appl Environ Microbiol. 2005;71(10):6418-22.

35. Kawahara M, Rikihisa Y, Lin Q, Isogai E, Tahara K, Itagaki A, Hiramitsu Y, Tajima T. Novel genetic variants of Anaplasma phagocytophilum, Anaplasma bovis, Anaplasma centrale, and a novel Ehrlichia sp. in wild deer and ticks on two major islands in Japan. Appl Environ Microbiol. 2006;72(2):1102-9.

\section{Submit your next manuscript to BioMed Central and we will help you at every step:}

- We accept pre-submission inquiries

- Our selector tool helps you to find the most relevant journal

- We provide round the clock customer support

- Convenient online submission

- Thorough peer review

- Inclusion in PubMed and all major indexing services

- Maximum visibility for your research

Submit your manuscript at www.biomedcentral.com/submit 